\title{
THE SPECIFICITY OF THE SABIN-FELDMAN DYE TEST WITH REFERENCE TO PROTOZOAL INFECTIONS
}

\author{
BY \\ C. KULASIRI \\ From the Department of Parasitology, London School of Hygiene and Tropical Medicine, London
}

(RECEIVED FOR PUBLICATION MARCH 12, 1960)

\begin{abstract}
Infections of Eimeria stiedae in normal and splenectomized rabbits and of Leishmania enriettii in normal rabbits and guinea-pigs and in splenectomized rabbits did not produce dye test antibodies. Guinea-pigs injected with Atoxoplasma sp. and rabbits immunized with cultures of Crithidia fasciculata were found to be negative for dye test antibodies. Mice which had been infected with Trypanosoma cruzi did not show dye test antibodies. All these animals, except one guinea-pig infected with Leishmania enriettii, were negative in the complement-fixation test for toxoplasmosis using the egg antigen. These findings are discussed in the light of previous reports.
\end{abstract}

Westphal and Knüttgen (1950) were the first to examine the specificity of the dye test (Sabin and Feldman, 1948) with reference to protozoal infections. They could not obtain a positive dye test in the sera of 14 patients suffering from tertian malaria or in the sera of two patients, one with amoebic dysentery and the other with mucocutaneous leishmaniasis. Westphal (see Mühlpfordt, 1951; Thalhammer, 1957) could not find dye test antibodies in infections of trypanosomes. However, Mühlpfordt (1951) was able to demonstrate dye test antibodies in rabbits, rats, and a golden hamster which had been experimentally infected with Sarcocystis. In sheep harbouring Sarcocystis tenella he found antibodies which were ascribed to the presence of these infections. Dye test antibodies were also detected in sheep infected with Sarcocystis tenella by Awad and Lainson (1954a, b) and by Awad (1954a) using his modified dye test. However, Moscovici (1953, 1954) could not find any antigenic relationship between Sarcocystis and Toxoplasma. Michalzik (1953) observed a high percentage of dye test antibodies in women harbouring infections of Trichomonas vaginalis. This was experimentally confirmed by Awad (1954b) in mice immunized with bacteriafree, living $T$. vaginalis. He also reported the presence of dye test antibodies in mice infected with Trypanosoma cruzi. Cathie (1955a, b) refuted some of these findings. Frenkel (1953) could not demonstrate any antigenic relationship between Toxoplasma and Besnoitia jellisoni. Since contradictory reports had been made on the production of dye test antibodies due to protozoal infections, it was felt necessary to undertake a detailed study to verify these observations.

\section{MATERIALS AND METHODS}

The following procedures were adopted throughout the study except where otherwise stated. A sample of blood was obtained from each experimental animal before infection. The bleeding of the experimental animals was carried out once a week, by cardiac puncture without any anaesthetic. About $8 \mathrm{ml}$. of blood was obtained at a time. It was allowed to clot at room temperature and the clot separated where necessary by means of a sterile platinum loop. The specimens were refrigerated overnight and centrifuged in the morning for 15 minutes at full speed, using an M.S.E. bench centrifuge. The separated serum was bottled in Bijou bottles and stored in the deep freeze at $-17^{\circ} \mathrm{C}$. till required for study. The period of storage was no longer than four months.

As accessory factor sera human sera were used from three donors who had been previously tested for this purpose and found suitable. The accessory factor sera were stored over dry ice and only thawed just before use to minimize the loss of the accessory factor content. The period of such storage never exceeded four months.

The Toxoplasma suspension was prepared by adding $0.1 \mathrm{ml}$. of a 1:500 solution of heparin in saline to each of the peritoneal exudates from albino mice 6 to 8 weeks old that had been injected three days previously with 1:10 suspension of peritoneal fluid (each high-power microscopic field containing about 15 to 25 parasites) from mice harbouring three-day infections of the " $\mathrm{RH}$ " strain of Toxoplasma gondii. Those suspensions showing a volume of 0.5 to $1.0 \mathrm{ml}$ were selected first. These were then examined under the microscope for adequate numbers of Toxoplasma. Only exudates which were free from bacterial contaminants and which showed over 100 Toxoplasma per high-power microscopic field were finally selected for use in the test. An aqueous solution of $0.25 \%$ methylene blue was used as the indicator and was prepared in the carbonate-borate buffer, as recom- 
mended by Sabin and Feldman (1948), a week earlier than required. This solution was used during the subsequent week and then discarded.

The freshly thawed test sera were inactivated for half an hour in a water-bath at $56^{\circ} \mathrm{C}$. Four-fold serial dilutions were carried out up to an original dilution of $1: 256$ using $0.85 \%$ saline as diluent. To 2 vol. of each of the serum dilutions were added 4 vol. of the freshly thawed accessory factor serum and 1 vol. of the heparinized Toxoplasma suspension. A suspension control was instituted for the Toxoplasma suspension from each mouse. This was prepared by adding 2 vol. of saline and $4 \mathrm{vol}$. of the accessory factor serum to $1 \mathrm{vol}$. of the Toxoplasma suspension. A known positive serum control was added to detect the presence of a sufficient amount of accessory factor in the accessory factor serum. The positive serum was obtained from a rabbit previously injected with the avirulent " RB25" strain of Toxoplasma. The contents of the tubes were mixed thoroughly by shaking the tube racks gently. The tubes were incubated for 45 minutes in a water-bath at $37^{\circ} \mathrm{C}$. At the end of this period 1 vol. of alkaline methylene blue was added to each tube and mixed thoroughly as before. The tubes were again incubated for a further five minutes. The readings were made immediately after removal from the water-bath. Whenever the readings could not be carried out immediately the tubes were refrigerated soon after removal from the water-bath. However, all tests were always read before the elapse of two hours after the addition of the methylene blue. A batch of results was accepted only if the accessory factor serum control showed less than $10 \%$ modification and the positive control worked faultlessly. The titre was taken as the highest original dilution showing more than $50 \%$ modification of the extracellular Toxoplasma.

The complement fixation test of Warren and Russ (1948) was also carried out on the sera with some of the modifications as adopted by Lainson (1955). The sera for the complement fixation test were inactivated for half an hour in a water-bath at $56^{\circ} \mathrm{C}$. They were serially diluted in two-fold dilutions up to a maximum dilution of $1: 16$, using $0.85 \%$ saline. One volume of antigen containing 4 units and 1 vol. of the complement diluted to contain 4 M.H.D. per volume were added to $1 \mathrm{vol}$. of each of the serum dilutions. An antigen control obtained from uninfected chick chorio-allantoic membranes and an anti-complement control were instituted for each serum and known positive and negative serum controls were added to each batch of tests. The contents of the tubes were mixed thoroughly by shaking the racks. They were incubated for one hour in a water-bath at $37^{\circ} \mathrm{C}$. At the end of this period 2 vol. of a sensitized sheep cell suspension prepared by mixing equal volumes of a haemolysin solution containing 6 M.H.D. of haemolysin and a $5 \%$ sheep cell suspension and incubating together for half an hour at $37^{\circ} \mathrm{C}$. were added. The contents of the tubes were mixed as before and reincubated for another half an hour. The results of the tests were read only if the controls had worked faultlessly by this time.

\section{EXPERIMENTS}

Experiment RBC: Dye Test Antibodies in Rabbits Infected with Eimeria stiedae

Twenty-four normal rabbits, 11-13 weeks old, were bled in two batches of 12 each. They were then fed witks a large quantity of sporulated oocysts of Eimeria stieda obtained from the faeces of infected rabbits. The oocysts were concentrated by salt flotation and sporulated if $\rho$ $2 \%$ sodium dichromate solution. The sodium dichromate was washed off by repeated centrifuging in water beforefeeding the rabbits. Weekly samples of blood werew drawn from the rabbits as before, up to a total of eigh? bleedings or the prior death of the rabbit. Samples of blood were also taken after death by cutting open the superior and inferior venae cavae in a few.

The tests were carried out as detailed earlier on inactivated and non-inactivated sera. Complement -6 fixation tests were carried out on inactivated sera.

Results.-These are set out in Table I. Two rabbitsc died at the first cardiac puncture. Fourteen rabbits died of coccidiosis by the third week, five rabbits by the fourth week, two rabbits by the fifth week, and ones rabbit survived the infection and was sacrificed later. Three rabbits did not show any dye test titres at all. Theo rest had dye test titres ranging from 1 to $1: 16$. The titreso fluctuated in the same rabbit between these limits. There was a slight difference in titre between the inactivatedo sera and the corresponding non-inactivated ones, buto this difference was negligible, being of the order of one dilution. All rabbits were negative for complement fixing $\overrightarrow{\widehat{O}}$ antibodies.

\section{Experiment RBCS: Dye Test Antibodies in Splenectomized Rabbits Infected with Eimeria stiedae}

Twenty-four rabbits, 10-12 weeks old, were splenecto-? mized using standard techniques. About four weeks 3 after the last splenectomy, they were bled in two batches: and fed with a smaller dose of sporulated oocysts of $E$. stiedae than in the previous experiment. Weekly samples of blood were drawn. Dye tests and complement음 fixation tests were carried out on inactivated sera. Dye tests were also carried out on non-inactivated sera.

Results.-The results are summarized in Table II. Two N rabbits died at the end of the seventh week. The rest survived the infection, probably due to being oldero rabbits than those in the previous experiment and also N being infected with a smaller dose. Two rabbits wereo completely negative in the dye test. The rest showed titres ranging from 1 to $1: 16$. As before there was a slight difference of titre in the results of the inactivated 0 and non-inactivated sera, but this was not significant. The complement fixation tests were negative.

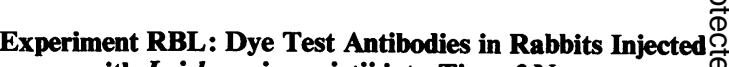 with Leishmania enrietii into Tips of Noses}

Twenty-four rabbits, 6-8 weeks old, were bled in two batches and injected with Leishmania enriettii obtained 8 from lesions of previously infected guinea-pigs. Weeklyo 
TABLE I

DYE TEST ANTIBODIES IN NORMAL RABBITS INFECTED WITH EIMERIA STIEDAE

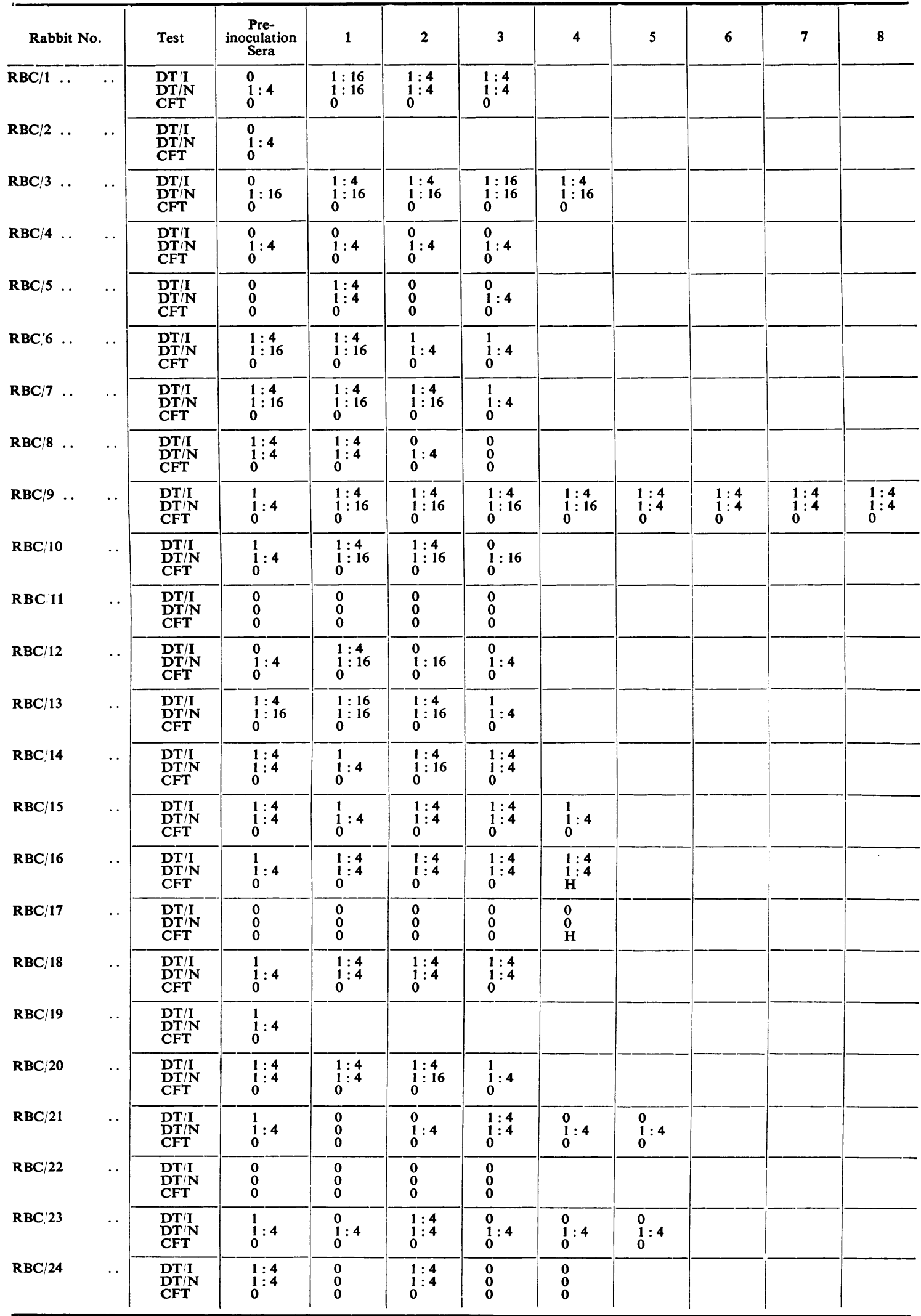




\begin{tabular}{|c|c|c|c|c|c|c|c|c|c|c|c|}
\hline Rabbit No & & Test & $\begin{array}{c}\text { Pre- } \\
\text { inoculation } \\
\text { Sera }\end{array}$ & 1 & 2 & 3 & 4 & 5 & 6 & 7 & 8 \\
\hline$\overline{R B C S} 1$ & $\ldots$ & $\begin{array}{l}\text { DT I } \\
\text { DT N } \\
\text { CFT }\end{array}$ & $\begin{array}{l}0 \\
1: 4 \\
0\end{array}$ & $\begin{array}{l}1 \\
1: 4 \\
0\end{array}$ & $\begin{array}{l}0 \\
1: 16 \\
0\end{array}$ & $\begin{array}{l}1: 4 \\
1: 16 \\
0\end{array}$ & $\begin{array}{l}1: 4 \\
1: 16 \\
0\end{array}$ & $\begin{array}{l}1: 4 \\
1: 4 \\
0\end{array}$ & $\begin{array}{l}1: 4 \\
1: 16 \\
0\end{array}$ & $\begin{array}{l}1: 4 \\
1: 4 \\
0\end{array}$ & \\
\hline RBCS 2 & .. & $\begin{array}{l}\text { DT I } \\
\text { DT N } \\
\text { CFT }\end{array}$ & $\begin{array}{l}1: 4 \\
1: 16 \\
0\end{array}$ & $\begin{array}{l}1: 4 \\
1: 4 \\
0\end{array}$ & $\begin{array}{l}1: 4 \\
1: 4 \\
0\end{array}$ & $\begin{array}{l}1: 4 \\
1: 4 \\
0\end{array}$ & $\begin{array}{l}1: 4 \\
1: 4 \\
0\end{array}$ & $\begin{array}{l}1: 4 \\
1: 4 \\
0\end{array}$ & $\begin{array}{l}1: 4 \\
1: 4 \\
0\end{array}$ & $\begin{array}{l}1: 4 \\
1: 4 \\
0\end{array}$ & $\begin{array}{l}1: 4 \\
1: 4 \\
0\end{array}$ \\
\hline RBCS 3 & .. & $\begin{array}{l}\text { DT I } \\
\text { DT N } \\
\text { CFT }\end{array}$ & $\begin{array}{l}1: 4 \\
1: 16 \\
0\end{array}$ & $\begin{array}{l}1: 4 \\
1: 16 \\
0\end{array}$ & $\begin{array}{l}1: 4 \\
1: 16 \\
0\end{array}$ & $\begin{array}{l}1: 4 \\
1: 16 \\
0\end{array}$ & $\begin{array}{l}1: 4 \\
1: 16 \\
0\end{array}$ & $\begin{array}{l}1: 4 \\
1: 4 \\
0\end{array}$ & $\begin{array}{l}1: 4 \\
1: 4 \\
0\end{array}$ & $\begin{array}{l}1: 4 \\
1: 4 \\
0\end{array}$ & $\begin{array}{l}1: 4 \\
1: 4 \\
0\end{array}$ \\
\hline RBCS 4 & .. & $\begin{array}{l}\text { DT I } \\
\text { DT N } \\
\text { CFT }\end{array}$ & $\begin{array}{l}0 \\
1: 4 \\
0\end{array}$ & $\begin{array}{l}1: 4 \\
1: 4 \\
0\end{array}$ & $\begin{array}{l}1: 4 \\
1: 4 \\
0\end{array}$ & $\begin{array}{l}1: 4 \\
1: 4 \\
0\end{array}$ & $\begin{array}{l}1: 4 \\
1: 16 \\
0\end{array}$ & $\begin{array}{l}1: 4 \\
1: 4 \\
0\end{array}$ & $\begin{array}{l}1: 4 \\
1: 4 \\
0\end{array}$ & $\begin{array}{l}1: 4 \\
1: 4 \\
0\end{array}$ & $\begin{array}{l}1: 4 \\
1: 4 \\
0\end{array}$ \\
\hline RBCS 5 & .. & $\begin{array}{l}\text { DT I I } \\
\text { DT } \\
\text { CFT }\end{array}$ & $\begin{array}{l}1: 4 \\
1: 4 \\
0\end{array}$ & $\begin{array}{l}1: 4 \\
1: 16 \\
0\end{array}$ & $\begin{array}{l}1: 16 \\
1: 16 \\
0\end{array}$ & $\begin{array}{l}1: 16 \\
1: 16 \\
0\end{array}$ & $\begin{array}{l}1: 16 \\
1: 16 \\
0\end{array}$ & $\begin{array}{l}1: 16 \\
1: 16 \\
0\end{array}$ & $\begin{array}{l}1: 4 \\
1: 16 \\
0\end{array}$ & $\begin{array}{l}1: 4 \\
1: 16 \\
0\end{array}$ & $\begin{array}{l}1: 4 \\
1: 16 \\
0\end{array}$ \\
\hline RBCS 6 & .. & $\begin{array}{l}\text { DT I } \\
\text { DT N } \\
\text { CFT }\end{array}$ & $\begin{array}{l}1: 4 \\
1: 16 \\
0\end{array}$ & $\begin{array}{l}1 \\
1: 16 \\
0\end{array}$ & $\begin{array}{l}1: 4 \\
1: 16 \\
0\end{array}$ & $\begin{array}{l}1: 4 \\
1: 16 \\
0\end{array}$ & $\begin{array}{l}1: 4 \\
1: 16 \\
0\end{array}$ & $\begin{array}{l}1: 4 \\
1: 4 \\
0\end{array}$ & $\begin{array}{l}1: 4 \\
1: 4 \\
0\end{array}$ & $\begin{array}{l}1: 4 \\
1: 4 \\
0\end{array}$ & $\begin{array}{l}1: 4 \\
1: 4 \\
0\end{array}$ \\
\hline RBCS 7 & .. & $\begin{array}{l}\text { DT I } \\
\text { DT N } \\
\text { CFT }\end{array}$ & $\begin{array}{l}0 \\
0 \\
0\end{array}$ & $\begin{array}{l}0 \\
0 \\
0\end{array}$ & $\begin{array}{l}0 \\
0 \\
0\end{array}$ & $\begin{array}{l}0 \\
0 \\
0\end{array}$ & $\begin{array}{l}0 \\
0 \\
0\end{array}$ & $\begin{array}{l}0 \\
0 \\
0\end{array}$ & $\begin{array}{l}0 \\
0 \\
0\end{array}$ & $\begin{array}{l}0 \\
0 \\
0\end{array}$ & $\begin{array}{l}0 \\
0 \\
0\end{array}$ \\
\hline RBCS 8 & .. & $\begin{array}{l}\text { DT I } \\
\text { DT N } \\
\text { CFT }\end{array}$ & $\begin{array}{l}0 \\
0 \\
0 \\
\end{array}$ & $\begin{array}{l}1: 4 \\
1: 4 \\
0\end{array}$ & $\begin{array}{l}1: 4 \\
1: 4 \\
0\end{array}$ & $\begin{array}{l}1: 4 \\
1: 4 \\
0\end{array}$ & $\begin{array}{l}1: 4 \\
1: 16 \\
0\end{array}$ & $\begin{array}{l}1: 4 \\
1: 16 \\
0\end{array}$ & $\begin{array}{l}1: 4 \\
1: 16 \\
0\end{array}$ & $\begin{array}{l}1: 4 \\
1: 16 \\
0\end{array}$ & $\begin{array}{l}1: 4 \\
1: 16 \\
0\end{array}$ \\
\hline RBCS 9 & .. & $\begin{array}{l}\text { DT I } \\
\text { DT N } \\
\text { CFT }\end{array}$ & $\begin{array}{l}0 \\
1: 4 \\
0\end{array}$ & $\begin{array}{l}0 \\
1: 16 \\
0\end{array}$ & $\begin{array}{l}1: 4 \\
1: 16 \\
0\end{array}$ & $\begin{array}{l}1: 4 \\
1: 16 \\
0\end{array}$ & $\begin{array}{l}1: 4 \\
1: 16 \\
0\end{array}$ & $\begin{array}{l}1: 4 \\
1: 16 \\
0\end{array}$ & $\begin{array}{l}1: 4 \\
1: 16 \\
0\end{array}$ & $\begin{array}{l}1: 4 \\
1: 16 \\
0\end{array}$ & $\begin{array}{l}1: 4 \\
1: 16 \\
0\end{array}$ \\
\hline RBCS 10 & .. & $\begin{array}{l}\text { DT I } \\
\text { DT N } \\
\text { CFT }\end{array}$ & $\begin{array}{l}0 \\
0 \\
0 \\
\end{array}$ & $\begin{array}{l}1: 4 \\
1: 16 \\
0\end{array}$ & $\begin{array}{l}1: 4 \\
1: 4 \\
0\end{array}$ & $\begin{array}{l}1: 4 \\
1: 4 \\
0\end{array}$ & $\begin{array}{l}1: 4 \\
1: 4 \\
0\end{array}$ & $\begin{array}{l}1: 4 \\
1: 4 \\
0\end{array}$ & $\begin{array}{l}1: 4 \\
1: 4 \\
0\end{array}$ & $\begin{array}{l}1: 4 \\
1: 4 \\
0\end{array}$ & $\begin{array}{l}1: 4 \\
1: 4 \\
0\end{array}$ \\
\hline RBCS 11 & .. & $\begin{array}{l}\text { DT I } \\
\text { DT N } \\
\text { CFT }\end{array}$ & $\begin{array}{l}0 \\
0 \\
0 \\
\end{array}$ & $\begin{array}{l}0 \\
1: 4 \\
0\end{array}$ & $\begin{array}{l}0 \\
1: 4 \\
0\end{array}$ & $\begin{array}{l}0 \\
1: 4 \\
0\end{array}$ & $\begin{array}{l}0 \\
0 \\
0 \\
\end{array}$ & $\begin{array}{l}0 \\
0 \\
0\end{array}$ & $\begin{array}{l}0 \\
1: 4 \\
0\end{array}$ & $\begin{array}{l}0 \\
1: 4 \\
0\end{array}$ & $\begin{array}{l}0 \\
1: 4 \\
0\end{array}$ \\
\hline RBCS 12 & .. & $\begin{array}{l}\text { DT I } \\
\text { DT N } \\
\text { CFT }\end{array}$ & $\begin{array}{l}0 \\
0 \\
0 \\
\end{array}$ & $\begin{array}{l}0 \\
0 \\
0\end{array}$ & $\begin{array}{l}1: 4 \\
1: 4 \\
0\end{array}$ & $\begin{array}{l}1: 4 \\
1: 4 \\
0\end{array}$ & $\begin{array}{l}1: 4 \\
1: 4 \\
0\end{array}$ & $\begin{array}{l}1: 4 \\
1: 4 \\
0\end{array}$ & $\begin{array}{l}1: 4 \\
1: 4 \\
0\end{array}$ & $\begin{array}{l}1: 4 \\
1: 4 \\
0\end{array}$ & $\begin{array}{l}1: 4 \\
1: 4 \\
0\end{array}$ \\
\hline RBCS 13 &.. & $\begin{array}{l}\text { DT I } \\
\text { DT N } \\
\text { CFT }\end{array}$ & $\begin{array}{l}1: 4 \\
1: 16 \\
0\end{array}$ & $\begin{array}{l}1 \\
1: 4 \\
0\end{array}$ & $\begin{array}{l}1: 16 \\
1: 16 \\
0\end{array}$ & $\begin{array}{l}1: 4 \\
1: 4 \\
0\end{array}$ & $\begin{array}{l}1: 4 \\
1: 16 \\
0\end{array}$ & $\begin{array}{l}1 \\
1: 4 \\
0\end{array}$ & $\begin{array}{l}1 \\
1: 4 \\
0\end{array}$ & $\begin{array}{l}1 \\
0\end{array}: 4$ & \\
\hline RBCS 14 & . & $\begin{array}{l}\text { DT I } \\
\text { DT N } \\
\text { CFT }\end{array}$ & $\begin{array}{l}1: 4 \\
1: 16 \\
0\end{array}$ & $\begin{array}{l}1: 4 \\
1: 16 \\
0\end{array}$ & $\begin{array}{l}1: 4 \\
1: 16 \\
0\end{array}$ & $\begin{array}{l}1: 4 \\
1: 16 \\
0\end{array}$ & $\begin{array}{l}1: 4 \\
1: 16 \\
0\end{array}$ & $\begin{array}{l}1: 4 \\
1: 4 \\
0\end{array}$ & $\begin{array}{l}1: 4 \\
1: 4 \\
0\end{array}$ & $\begin{array}{l}1: 4 \\
1: 4 \\
0 \\
\end{array}$ & $\begin{array}{l}1: 4 \\
1: 4 \\
0 \\
\end{array}$ \\
\hline RBCS 15 &.. & $\begin{array}{l}\text { DT I } \\
\text { DT N } \\
\text { CFT }\end{array}$ & $\begin{array}{l}1: 4 \\
1: 16 \\
0\end{array}$ & $\begin{array}{l}1: 4 \\
1: 16 \\
0\end{array}$ & $\begin{array}{l}1: 4 \\
1: 16 \\
0\end{array}$ & $\begin{array}{l}1: 4 \\
1: 16 \\
0\end{array}$ & $\begin{array}{l}1: 4 \\
1: 4 \\
0\end{array}$ & $\begin{array}{l}1: 4 \\
1: 4 \\
0\end{array}$ & $\begin{array}{l}1: 4 \\
1: 4 \\
0\end{array}$ & $\begin{array}{l}1: 4 \\
1: 4 \\
0\end{array}$ & $\begin{array}{l}1: 4 \\
1: 4 \\
0\end{array}$ \\
\hline RBCS 16 & .. & $\begin{array}{l}\text { DT I } \\
\text { DT N } \\
\text { CFT }\end{array}$ & $\begin{array}{l}1 \\
1: 4 \\
0\end{array}$ & $\begin{array}{l}1: 4 \\
1: 16 \\
0\end{array}$ & $\begin{array}{l}1: 4 \\
1: 16 \\
0\end{array}$ & $\begin{array}{l}1: 4 \\
1: 16 \\
0\end{array}$ & $\begin{array}{l}0 \\
1: 16 \\
0\end{array}$ & $\begin{array}{l}1: 4 \\
1: 16 \\
0\end{array}$ & $\begin{array}{l}1: 4 \\
1: 16 \\
0\end{array}$ & $\begin{array}{l}1: 4 \\
1: 16 \\
0\end{array}$ & $\begin{array}{l}1: 4 \\
1: 169 \\
0\end{array}$ \\
\hline RBCS 17 & .. & $\begin{array}{l}\text { DT I } \\
\text { DT N } \\
\text { CFT }\end{array}$ & $\begin{array}{l}0 \\
0 \\
0 \\
\end{array}$ & $\begin{array}{l}0 \\
0 \\
0 \\
\end{array}$ & $\begin{array}{l}0 \\
0 \\
0 \\
\end{array}$ & $\begin{array}{l}0 \\
0 \\
0\end{array}$ & $\begin{array}{l}0 \\
0 \\
0 \\
\end{array}$ & $\begin{array}{l}0 \\
0 \\
0\end{array}$ & $\begin{array}{l}0 \\
0 \\
0\end{array}$ & $\begin{array}{l}0 \\
0 \\
0\end{array}$ & $\begin{array}{l}0 \\
0 \\
0 \\
\end{array}$ \\
\hline RBCS 18 & .. & $\begin{array}{l}\text { DT I } \\
\text { DT N } \\
\text { CFT }\end{array}$ & $\begin{array}{l}0 \\
1: 4 \\
0 \\
\end{array}$ & $\begin{array}{l}1: 4 \\
1: 4 \\
0\end{array}$ & $\begin{array}{l}0 \\
1: 4 \\
0\end{array}$ & $\begin{array}{l}1: 4 \\
1: 4 \\
0\end{array}$ & $\begin{array}{l}0 \\
1: 4 \\
0\end{array}$ & $\begin{array}{l}1: 4 \\
1: 4 \\
0\end{array}$ & $\begin{array}{l}1: 4 \\
1: 4 \\
0\end{array}$ & $\begin{array}{l}1: 4 \\
1: 4 \\
0\end{array}$ & $\begin{array}{ll}1: 4 & n \\
1: 4 & 8 \\
0 & \end{array}$ \\
\hline RBCS 19 & . & $\begin{array}{l}\text { DT I } \\
\text { DT N } \\
\text { CFT }\end{array}$ & $\begin{array}{l}0 \\
0 \\
0\end{array}$ & $\begin{array}{l}0 \\
0 \\
0 \\
\end{array}$ & $\begin{array}{l}1 \\
1: 4 \\
0\end{array}$ & $\begin{array}{l}1: 4 \\
1: 16 \\
0\end{array}$ & $\begin{array}{l}1: 4 \\
1: 16 \\
0\end{array}$ & $\begin{array}{l}1: 4 \\
1: 4 \\
0\end{array}$ & $\begin{array}{l}1: 4 \\
1: 4 \\
0\end{array}$ & $\begin{array}{l}1: 4 \\
1: 16 \\
0\end{array}$ & $\begin{array}{l}1: 48 \\
1: 16 \frac{1}{c} \\
0\end{array}$ \\
\hline RBCS 20 & . & $\begin{array}{l}\text { DT I } \\
\text { DT N } \\
\text { CFT }\end{array}$ & $\begin{array}{l}1: 4 \\
1: 16 \\
0\end{array}$ & $\begin{array}{l}1: 4 \\
1: 16 \\
0\end{array}$ & $\begin{array}{l}1: 4 \\
1: 4 \\
0\end{array}$ & $\begin{array}{l}1: 4 \\
1: 16 \\
0\end{array}$ & $\begin{array}{l}1: 16 \\
1: 16 \\
0\end{array}$ & $\begin{array}{l}1: 16 \\
1: 16 \\
0 \\
\end{array}$ & $\begin{array}{l}1: 16 \\
1: 16 \\
0\end{array}$ & $\begin{array}{l}1: 16 \\
1: 16 \\
0\end{array}$ & $\begin{array}{l}1: 16 c \\
1: 16 c \\
0\end{array}$ \\
\hline RBCS 21 & . & $\begin{array}{l}\text { DT I } \\
\text { DT N } \\
\text { CFT }\end{array}$ & $\begin{array}{l}1: 4 \\
1: 4 \\
0\end{array}$ & $\begin{array}{l}1 \\
0\end{array}$ & $\begin{array}{l}1 \\
0 \\
0\end{array}$ & $\begin{array}{l}1: 4 \\
1: 4 \\
0\end{array}$ & $\begin{array}{l}1: 4 \\
1: 4 \\
0\end{array}$ & $\begin{array}{l}1: 4 \\
1: 4 \\
0\end{array}$ & $\begin{array}{l}1: 4 \\
1: 4 \\
0\end{array}$ & $\begin{array}{l}1: 4 \\
1: 4 \\
0\end{array}$ & $\begin{array}{l}1: 4 \\
1: 4 \\
0 \\
\end{array}$ \\
\hline RBCS 22 & .. & $\begin{array}{l}\text { DT I } \\
\text { DT N } \\
\text { CFT }\end{array}$ & $\begin{array}{l}0 \\
1: 16 \\
0\end{array}$ & $\begin{array}{l}0 \\
1: 4 \\
0\end{array}$ & $\begin{array}{l}1: 4 \\
1: 16 \\
0\end{array}$ & $\begin{array}{l}1: 4 \\
1: 16 \\
0\end{array}$ & $\begin{array}{l}1: 4 \\
1: 4 \\
0\end{array}$ & $\begin{array}{l}1: 4 \\
1: 16 \\
0\end{array}$ & $\begin{array}{l}1: 4 \\
1: 16 \\
0\end{array}$ & $\begin{array}{l}1: 4 \\
1: 16 \\
0\end{array}$ & $\begin{array}{l}1: 4 \pi \\
1: 16 \frac{\pi}{2} \\
0\end{array}$ \\
\hline RBCS 23 & .. & $\begin{array}{l}\text { DT I } \\
\text { DT N } \\
\text { CFT }\end{array}$ & $\begin{array}{l}1: 4 \\
1: 4 \\
0\end{array}$ & $\begin{array}{l}1: 4 \\
1: 16 \\
0\end{array}$ & $\begin{array}{l}1: 4 \\
1: 16 \\
0\end{array}$ & $\begin{array}{l}1: 16 \\
1: 16 \\
0\end{array}$ & $\begin{array}{l}1: 16 \\
1: 16 \\
0\end{array}$ & $\begin{array}{l}1: 16 \\
1: 16 \\
0\end{array}$ & $\begin{array}{l}1: 16 \\
1: 16 \\
0\end{array}$ & $\begin{array}{l}1: 16 \\
1: 16 \\
0\end{array}$ & $\begin{array}{l}1: 16 \\
1: 16 \\
0\end{array}$ \\
\hline RBCS 24 & .. & $\begin{array}{l}\text { DT I } \\
\text { DT N } \\
\text { CFT }\end{array}$ & $\begin{array}{l}0 \\
1: 4 \\
0\end{array}$ & $\begin{array}{l}0 \\
1: 16 \\
0\end{array}$ & $\begin{array}{l}1: 16 \\
1: 16 \\
0\end{array}$ & $\begin{array}{l}1: 16 \\
1: 16 \\
0\end{array}$ & $\begin{array}{l}1: 4 \\
1: 16 \\
0\end{array}$ & $\begin{array}{l}1: 4 \\
1: 16 \\
0\end{array}$ & $\begin{array}{l}1: 4 \\
1: 16 \\
0\end{array}$ & $\begin{array}{l}1: 4 \\
1: 16 \\
0\end{array}$ & $\begin{array}{l}1: 4 \bar{\zeta} \\
1: 16 \\
0\end{array}$ \\
\hline
\end{tabular}


samples of blood were obtained. Dye tests were carried out on inactivated sera only. Complement fixation tests were also carried out.

Results.-The results are set out in Table III. None of the rabbits was infected with $L$. enriettii macroscopically. One rabbit died by the end of the first week, one by the end of the second week, two by the end of the seventh week, and the rest were all sacrificed at the end of the eighth week. Four rabbits were completely negative of any antibodies. The rest had dye test titres between 1 and 1:4. They did not show any change in their titres.

\section{Experiment RBLS: Dye Test Antibodies in Splenectomized Rabbits Injected with Leishmania enriettii into Tips of Noses}

Twenty rabbits, 6-8 weeks old, were splenectomized as before. About three weeks after the last splenectomy, they were bled and $L$. enriettii, obtained from lesions of previously infected guinea-pigs, were injected into the tips of their noses. Dye tests and complement fixation tests were carried out on inactivated sera obtained weekly.

Results.-These are summarized in Table IV. From this group of rabbits, six (RBLS/3, RBLS/8, RBLS/9, $\mathrm{RBLS} / 13, \mathrm{RBLS} / 16$, and RBLS/18) were macroscopically infected with $L$. enriettii which was later confirmed microscopically. One rabbit died at the end of the first week, one rabbit at the end of the sixth week, and two at the end of the seventh week. The rest were kept under observation for another six weeks after the last bleeding at the end of the eighth week. One of the rabbits was completely negative for the dye tests. Once again the titres ranged from 1 to $1: 16$ with negative complement fixation tests. One of the rabbits which showed a titre in the undiluted serum suddenly increased its titre to $1: 16$ and remained at this value. This change is not considered as significant.

\section{Experiment GPL: Dye Test Antibodies in Guinea-pigs Infected with Leishmania enriettii}

Six guinea-pigs which were previously bled by cardiac puncture were injected with $L$. enriettii into the tips of their noses. At the end of the seventh week the guineapigs were bled. Eight weeks later they were bled for the last time. Dye tests and complement fixation tests were carried out on the inactivated sera.

Results.-The guinea-pig GPL/1 died three weeks after the second bleeding, and the guinea-pig GPL/4 four weeks after the second bleeding. All the dye tests and the complement fixation tests were negative except in the guinea-pig GPL/1, which showed a dye test titre of $1: 256$ and complement fixation titres of $1: 16$ in the pre-infection serum and the post-infection serum. Unfortunately the dye tests and complement fixation tests had not been carried out by this time and hence the brain of the guinea-pig could not be passaged into mice

TABLE III

DYE TEST ANTIBODIES IN RABBITS INJECTED WITH LEISHMANIA ENRIETTII INTO TIPS OF NOSES

\begin{tabular}{|c|c|c|c|c|c|c|c|c|c|c|}
\hline Rabbit No. & Test & $\begin{array}{c}\text { Pre- } \\
\text { inoculation } \\
\text { Sera }\end{array}$ & 1 & 2 & 3 & 4 & 5 & 6 & 7 & 8 \\
\hline 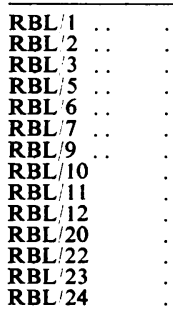 & $\begin{array}{l}\text { DT } \\
\text { CFT }\end{array}$ & $\begin{array}{l}1: 4 \\
0\end{array}$ & $\begin{array}{l}1: 4 \\
0\end{array}$ & $\begin{array}{l}1: 4 \\
0\end{array}$ & $\begin{array}{l}1: 4 \\
0\end{array}$ & $\begin{array}{l}1: 4 \\
0\end{array}$ & $\begin{array}{l}1: 4 \\
0\end{array}$ & $1: 4$. & $\begin{array}{l}1: 4 \\
0\end{array}$ & $1: 4$ \\
\hline RBL $4 \ldots$ & $\begin{array}{l}\text { DT } \\
\text { CFT }\end{array}$ & $1: 4$ & $1: 4$ & $1: 4$ & $1: 4$ & $1: 4$ & $1: 4$ & $1: 4$ & $1: 4$ & \\
\hline $\begin{array}{l:l}\text { RBL } & 8 \\
\text { RBL } & 16 \\
\text { RBL } & 17 \\
\text { RBL } & 18\end{array}$ & $\begin{array}{l}\text { DT } \\
\text { CFT }\end{array}$ & $\begin{array}{l}0 \\
0\end{array}$ & $\begin{array}{l}0 \\
0\end{array}$ & $\begin{array}{l}0 \\
0\end{array}$ & $\begin{array}{l}0 \\
0\end{array}$ & $\begin{array}{l}0 \\
0\end{array}$ & $\begin{array}{l}0 \\
0\end{array}$ & $\begin{array}{l}0 \\
0\end{array}$ & $\begin{array}{l}0 \\
0\end{array}$ & $\begin{array}{l}0 \\
0\end{array}$ \\
\hline RBL 13 & $\begin{array}{l}\text { DT } \\
\text { CFT }\end{array}$ & $1: 4$ & & & & & & & & \\
\hline $\begin{array}{l:l}\text { RBL } & 14 \\
\text { RBL } & 15\end{array}$ & $\begin{array}{l}\text { DT } \\
\text { CFT }\end{array}$ & $1: 4$ & $\begin{array}{l}1: 4 \\
0\end{array}$ & $1: 4$ & $1: 4$ & $\begin{array}{l}0 \\
0\end{array}$ & $\begin{array}{l}0 \\
0\end{array}$ & $\begin{array}{l}\mathbf{0} \\
\mathbf{0}\end{array}$ & $\begin{array}{l}0 \\
0\end{array}$ & $\begin{array}{l}0 \\
0\end{array}$ \\
\hline$\overline{\text { RBL'19 }}$ & $\begin{array}{l}\text { DT } \\
\text { CFT }\end{array}$ & $\begin{array}{l}\mathbf{0} \\
\mathbf{0}\end{array}$ & $1: 4$ & $1: 4$ & & & & & & \\
\hline$\overline{R B L}$ '21 & $\begin{array}{l}\text { DT } \\
\text { CFT }\end{array}$ & $\begin{array}{l}0 \\
0\end{array}$ & $1: 4$ & $1: 4$ & $1: 4$ & $1: 4$ & $\begin{array}{l}1: 4 \\
0\end{array}$ & $1: 4$ & $1: 4$ & $1: 4$ \\
\hline
\end{tabular}

$I=$ inactivated sera. $\mathrm{N}=$ non-inactivated sera. $\mathrm{H}=$ haemolysed sera. $1,2,3$, etc. $=$ weekly sera obtained after infection. $\mathrm{DT}=$ dye test . CFT $=$ complement fixation test. 
TABLE IV

DYE TEST ANTIBODIES IN SPLENECTOMIZED RABBITS INJECTED WITH LEISHMANIA ENRIETTII INTO TIPS OF NOSES

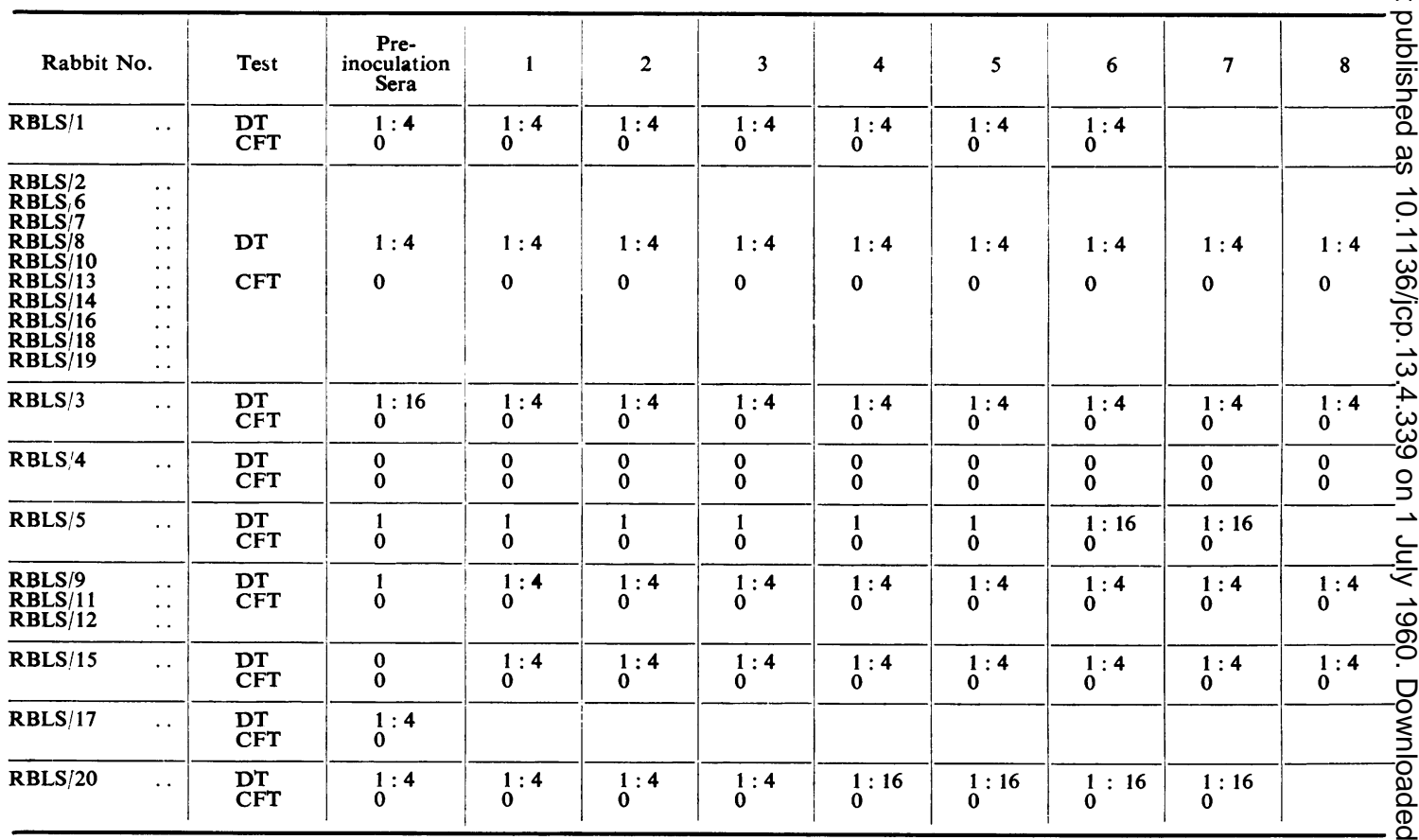

I= inactivated sera. $\quad \mathbf{N}=$ non-inactivated sera. $\quad \mathbf{H}=$ haemolysed sera. $\quad 1,2,3$, etc. = weekly sera obtained after infection. $\quad$ DT $=$ dye test. $\overrightarrow{\bar{O}}$ $\mathrm{CFT}=$ complement fixation test.

for the isolation of Toxoplasma. This guinea-pig was, however, considered to harbour a Toxoplasma infection or have had some contact with it previously.

\section{Experiment RBCR: Dye Test Antibodies in Rabbits Intraperitoneally Injected with Crithidia fasciculata}

A culture of Crithidia fasciculata was isolated by Professor P. C. C. Garnham from a Taeniorhynchus mosquito. This was maintained in the NNN medium with penicillin and streptomycin by Mr. P. E. Nesbitt, who also prepared the inocula used in this experiment. Two rabbits, previously bled, were injected intraperitoneally with $1 \mathrm{ml}$. of the NNN medium containing the parasites. One week later another injection was given. At the end of the second week from the last inoculation the rabbits were bled. They were again injected with the NNN medium containing the parasites four weeks after the post-inoculation bleeding and once again after a week. At the end of two weeks after the last injection the rabbits were bled for the last time. Dye and complement fixation tests were carried out as before on inactivated sera.

Results.-One of the rabbits was completely negative for the dye test and the complement fixation test antibodies. The other had a dye test titre of $1: 4$ in all three specimens of sera. The complement fixation tests were negative.
Experiment GPA: Dye Test Antibodies in Guinea-pigs Intraperitoneally Injected with Atoxoplasma sp.

The Atoxoplasma was isolated from sparrows at The $\overline{0}$ Winches Farm, St. Albans, Herts., by Dr. R. Lainson, who supplied me with the spleens of birds infected with 3 . the parasite. Six guinea-pigs which were previously bled were injected with ground spleens of four sparrows which were microscopically positive for Atoxoplasma. Twelve weeks later they were given another injection of ground 윽 spleens containing the parasites. Twenty-four weeks after $D$ the first injections the guinea-pigs were bled by cardiac puncture. Dye tests and complement fixation tests were $\bar{N}$ carried out on these sera after inactivation.

Results.-All the dye tests and the complement fixation $\mathrm{N}$ tests were negative.

\section{Experiment MST: Dye Test Antibodies in Mice Infected with Trypanosoma cruzi}

Six mice which had been infected about 12 weeks pre- $\stackrel{\infty}{+}$ viously with Trypanosoma cruzi were passed on to me by Mr. W. Cooper. All of them had been microscopically positive for $T$. cruzi at some time or other. The mice $\mathbb{D}$ were sacrificed and bled from the heart. Dye tests and $\mathbb{D}$ complement fixation tests were carried out on these sera

Results.-All the mice were completely free from dye test and complement fixing antibodies for Toxoplasma. 


\section{DISCUSSION}

Westphal and Knüttgen (1950) obtained negative dye tests in 14 cases of tertian malaria. Similarly, Awad and Lainson (1954b) could not demonstrate dye test antibodies in a rhesus monkey infected with Plasmodium cynomolgi. Jacobs (1956) did not obtain definite evidence for the production of dye test antibodies in rats infected with Plasmodium berghei or in chickens infected with Plasmodium gallinaceum. Cathie (1957) obtained three dye test positives in 76 sera from patients of all ages and with all stages of malaria from Uganda. Since the complement fixation test with the egg antigen was negative, he considered this of no importance. Similarly, Beverley (1957) obtained $35 \%$ of 85 cases of malaria in the Forces positive to the dye test at a titre of $1: 4$ (final dilution) and $2.5 \%$ positive in the complement fixation test. $\mathrm{He}$ also did not consider this significant as he expected $100 \%$ overlap if there was any antigenic relationship. Of the sera of three patients who were examined by Beverley (1957) before and after experimental infection with Plasmodium vivax, one serum remained negative and the other two weakly positive before and after infection. Jacobs (1956) could not obtain positive dye tests in a large number of squirrels naturally infected with a species of Hepatozoon or in chicks infected with Eimeria tenella.

Mühlpfordt (1951) obtained positive dye tests in guinea-pigs, rats, and a hamster infected with Sarcocystis experimentally. The guinea-pigs, which were previously negative, developed titres of $1: 25$ and 1:50 while the rats, which were also negative, developed titres of $1: 200$ and $1: 400$. These titres were maintained at the end of eight and twelve weeks respectively. In the hamster the titre was $1: 25$ at four weeks and 1:200 at seven weeks. In a survey of dye test antibodies in 45 sheep, Mühlpfordt (1951) found 12 sheep with positive dye tests and macroscopic infections of sarcosporidia, 10 sheep with positive dye tests but no macroscopic infections of sarcosporidia, and 23 sheep with negative dye tests and no macroscopic infections. Since macroscopic examination of throat muscles would not show incipient infections and as the rate of infection otherwise was low compared with figures given by other authors, Mühlpfordt (1951) assumed that those showing a dye test titre also harboured Sarcocystis infections. Finally, in a guinea-pig harbouring a macroscopic infection of Sarcocystis, he obtained a positive dye test titre of $1: 50$. On this evidence he assumed a cross reaction between Sarcocystis and Toxoplasma. Awad and Lainson (1954a, b) obtained similar results in sheep infected with sarcosporidia. Of 19 sheep positive for Sarcocystis, eight showed dye test titres, while out of six sheep negative for sarcosporidia, three showed dye test titres (Awad and Lainson, 1954b). Six lambs, which were negative in the complement fixation tests using both Toxoplasma and Sarcocystis antigens, were also negative in the dye test. Therefore Awad and Lainson $(1954 a, b)$ assumed the presence of a cross reaction between Toxoplasma and Sarcocystis. Using the spores of Sarcocystis tenella instead of Toxoplasma, Awad (1954a) was able to demonstrate dye test antibodies in three human sera showing dye test antibodies by the classical method, in another human serum which was negative by the usual method, and in three guinea-pigs which were experimentally infected with Toxoplasma. On the other hand, Moscovici (1953, 1954) could not demonstrate experimentally a serological affinity of a high grade between Toxoplasma and Sarcocystis. Of 45 sheep, 12 of which were definitely infected with Sarcocystis tenella, 22 showed positive reactions with titres between 1:4 and 1:10 (Moscovici, 1954). Guineapigs and rabbits, immunized with formalin-killed as well as living sarcosporidia, did not show any dye test antibodies, although they showed complementfixing antibodies with Sarcocystis antigen. Reid and Manning (1956) obtained positive dye tests, mostly to very high titres, in all of 36 sheep, most of which they assumed to be infected with sarcosporidia, but they could not obtain any positive complementfixation tests. Since these positives were obtained in the presence of accessory factor, they suggested that these were not due to non-specific factors. The isolation of Toxoplasma from placentae of sheep made the significance of these results uncertain to them. Cathie (1957) in a dye test survey among 69 sheep observed 54 to give a positive dye test with titres up to $1: 16$. However, after inactivation 20 sera turned out to be completely negative. On investigating 116 human sera containing both positives and negatives with the dye test using the modification of Awad (1954a), Cathie (1957) found all to give a positive dye test. Many of these sera retained this property of modifying sarcospores even after inactivation by heat. On further investigation Cathie and Cecil (1957) noticed that in 23 human sera, a third of which gave a positive dye test with Toxoplasma, all were giving a positive dye test at $1: 4$ dilution using sarcospores. Ninety-three other sera were also found to contain dye test antibodies using the test of Awad (1954a). On repeating the test with sarcospores and the previous 23 sera after inactivation at $56^{\circ} \mathrm{C}$. for 30 minutes, they became negative. On the other hand, Cathie and Cecil (1957) found, in a guinea-pig experimentally immunized with Sarcocystis complement-fixing antigen, that inactivation did not completely remove the antibodies detected 
with the use of sarcospores but retained them in a much lowered titre of $1: 4$, although no accessory factor was supplied at all, thus demonstrating conclusively that this substance present in the immunized guinea-pig was not the dye test antibodies as defined by Sabin and Feldman. In 10 sheep selected at random, Cathie and Cecil (1957) found six positive in the complement-fixation test using Sarcocystis antigen, and all 10 positive in the dye test with sarcospores. However, on inactivating these sera, this ability to modify fresh sarcospores was lost. On the other hand, tests carried out using old sarcospores and human sera showed that inactivating the sera had no effect in the modification as all sera showed positives whether inactivated or not. Varela, Roch, and Vázquez (1955) studied the sera of eight Rattus norvegicus with Sarcocystis and found three of them positive with the dye test at a titre of $1: 32$. Of two $R$. norvegicus with sarcosporidia, Trichinella and filaria, one rat showed a dye test positive at 1:32. Jacobs (1956) found three rhesus monkeys infected with Sarcocystis dye test negative while Sabin (see Jacobs, 1956) found negative dye tests in some cynomolgus monkeys with Sarcocystis infections. Thalhammer (1957) could not demonstrate dye test antibodies in rabbits suffering from sarcosporidiosis. Bakal (see van Thiel, 1958) found Sarcocystis hirsuta in hearts of cattle which were dye test positive as well as dye test negative. Awad and Lainson (1954b) reported a human case of sarcosporidiosis which was completely dye test negative. Another case, which was also completely negative, was cited by Beverley, Beattie, and Roseman (1954) and by Beverley (1957). The work of Mühlpfordt (1951), Awad and Lainson (1954a, b), and Reid and Manning (1956) suggests the possibility of cross reactions in infections of Sarcocystis. On the other hand, Moscovici $(1953,1954)$ could not obtain any serological relationship between Sarcocystis and Toxoplasma. However, Moscovici (1954) also could not obtain positive dye tests in rats, rabbits, and guinea-pigs infected with Toxoplasma living as well as killed. The results of Cathie (1957) and Cathie and Cecil (1957) throw some light on this problem. The complete removal of the dye test antibodies found in the sera of sheep after inactivation shows that there are some factors other than the dye test antibodies which are known to be heat resistant. These may be the factors involved in the observations of Mühlpfordt (1951) and Awad and Lainson (1954a, b). The presence of factors capable of modifying sarcospores even in the absence of the accessory factor invalidates the modification of the dye test suggested by Awad (1954a). The results obtained by Reid and Manning (1956) could be due to the same phenomenon observed by
Cathie (1957). I uman cases of sarcosporidiosis cited by Awad and Lainson (1954b), Beverley et al. (1954), and Beverley (1957) give additional support. All subsequent workers unanimously agree that Sarcocystis infections do not elaborate dye test antibodies.

Frenkel (1953) found Besnoitia jellisoni and Toxoplasma to be immunologically distinct. Jacobs (1956) found in some instances of rabbits infected with $B$. jellisoni dye test titres up to $1: 16$. However, he believed them not to have a cross immunity. Rats harbouring Encephalitozoon in the brain were found by Jacobs (1956) to show low dye test titres of 1 or $1: 4$. He suggested the possibility of cross reactions at low titres between Toxoplasma and Encephalitozoon. Considering the low titres reported by Jacobs (1956), this could be regarded as insignificant.

Michalzik (1953) obtained $64 \%$ dye test positives with a titre of $1: 25$ or higher in 50 adult women suffering from Trichomonas vaginalis. On the ground that this represented a higher percentage of positive dye tests than those reported by other German authors, he assumed that Trichomonas infections caused the formation of dye test antibodies. Cathie (1955a) obtained positive dye tests in adult women suffering from Trichomonas vaginitis. The sera of four adult men with Trichomonas infections had negative dye tests. However, Cathie (1955b) found similar proportions of dye test positives and negatives among comparable groups of women suffering from vaginitis and those free from it. On this ground he concluded that Trichomonas infections did not affect the dye test. Reid and Manning (1956) obtained three positive dye tests in seven patients with Trichomonas vaginitis. After studying with the dye test 132 women who were microscopically positive for $T$. vaginalis and 110 women microscopically negative for it, Piekarski, Saathoff, and Korte (1957) concluded that Trichomonas infections did not produce dye test antibodies. Borgen and Björnstad (1957) found 13 women suffering from severe trichomonad vaginitis for over six months to be completely negative, except one who showed a positive dye test in the undiluted serum. Beverley (1957) found $54 \%$ of 46 cases of Trichomonas infection in the Sheffield region to give a positive reaction at a titre of $1: 4$ (final dilution). In rabbits and mice experimentally immunized with living Trichomonas cultures, Awad (1954b) obtained high titres of dye test antibodies. The rabbits were initially dye test negative. The control mice used were free of dye test antibodies. On this basis Awad (1954b) concluded that Trichomonas vaginalis could produce antibodies detectable by the dye test. 
Piekarski et al. (1957) repeated the experimental studies of Awad (1954b). Of the 15 rabbits used in the study, 11 showed positive dye test titres between $1: 16$ and 1:256 in the pre-inoculation sera. After repeated inoculations of Trichomonas vaginalis from cultures, no essential differences in titres of the dye test and the complement-fixation test for toxoplasmosis were observed, although the inoculated animals showed strong complement-fixation tests with Trichomonas antigen. However, two rabbits which were negative at first became feebly positive with a titre of 1:4 in the dye test. Saathoff (see Piekarski et al., 1957) obtained similar results with mice injected intraperitoneally with Trichomonas vaginalis. Borgen and Björnstad (1957) did not find any dye test antibodies in 10 mice inoculated with living trichomonads. Similar experiments in rabbits by Bakal (see van Thiel, 1958) gave identical results in the dye test although an increase in the agglutination titre was observed. The claim of Michalzik (1953) that Trichomonas vaginalis infections caused the production of dye test antibodies could be dismissed as his control groups were not similar. This is clearly revealed by the results of the surveys obtained by Cathie (1955a, b), Reid and Manning (1956), Piekarski et al. (1957), Borgen and Björnstad (1957), and Beverley (1957). However, the experimental studies of Awad (1954b) cannot be dismissed so lightly. On the other hand the repetition of this work by Piekarski et al. (1957), Saathoff (see Piekarski et al. (1957), Borgen and Björnstad (1957), and Bakal (see van Thiel, 1958) does not support the observations of Awad (1954b). No explanations could be given as to the results obtained by Awad (1954b).

According to Westphal (see Mühlpfordt, 1951; Thalhammer, 1957), no dye test antibodies were built by trypanosomes. Awad (1954b) obtained titres up to 1:512 in four mice previously infected with Trypanosoma cruzi while the control mice were negative. Of 16 albino rats infected with $T$. cruzi, Varela et al. (1955) obtained dye test titres of $1: 32$ in 13 rats. However, Jacobs (1956) could not obtain serological reactions between Trypanosoma cruzi and Toxoplasma in infected rats. Cathie (1957) observed six out of 15 sera from cases of Chagas' disease to show a positive dye test at low titres which he regarded as insignificant. Bakal (see van Thiel, 1958) could not obtain positive results in animals inoculated with $T$. cruzi, although she was able to establish the infection in the peripheral blood of the inoculated animals. Grönroos and Salminen (1955) could not obtain a correlation between the dye test results and the presence of Trypanosoma lewisi in the blood in 108 wild rats, Rattus norvegicus. Cathie
(1957) found only two of 70 sera from patients suffering from infections of Trypanosoma gambiense to give dye test titres of $1: 16$ or lower. Beverley (1957) found $36 \%$ of 17 sera from trypanosome infections from Uganda and Nigeria to give positive dye test at a titre of 1:4 (final dilution) which was not regarded as of importance. Saathoff (see Piekarski, 1958) found that mice and guinea-pigs experimentally infected with Trypanosoma cruzi and guinea-pigs infected with $T$. evansi were completely free from dye test antibodies although most of them showed infections with these agents. Bakal (see van Thiel, 1958) could not obtain positive tests in experimental animals infected with Trypanosoma rhodesiense. Westphal and Knüttgen (1950) did not observe any dye test antibodies in a case of mucocutaneous leishmaniasis. Thus no cross reactions with the dye test were observed in infections with trypanosomes by Westphal (see Mühlpfordt, 1951; Thalhammer, 1957), Grönroos and Salminen (1955), Cathie (1957), and Beverley (1957). Once again the claim of Awad (1954b) could not be substantiated by the experimental work of Saathoff (see Piekarski, 1958) and Bakal (see van Thiel, 1958), although the work of Varela et al. (1955) confirmed Awad's findings. In the present study no dye test antibodies were detected in mice which had been infected with Trypanosoma cruzi. In spite of the contradictory claims of Awad (1954b) and Varela et al. (1955), the consensus of opinion points to the specificity of the dye test with regard to trypanosome infections.

Varela et al. (1955) found six rats positive in the dye test with a titre of $1: 32$ from 50 rats harbouring intestinal flagellates. Westphal and Knüttgen (1950) could not detect the presence of dye test antibodies in a case of amoebic dysentery.

Considering an increasing titre as the criterion for the production of dye test antibodies, no such phenomenon was observed in the experimental work undertaken in this study although a fluctuation in the titres was observed in rabbits infected with Eimeria stiedae. A titre over 1:16 was never recorded. This fluctuation could be attributed to the severe necrosis and fibrosis seen in the livers of these rabbits. Further, repeated weekly withdrawal of blood could be significant in animals suffering severe necrosis of the liver. Hence such a fluctuation should be expected as normal. Practically no variations in the titres were observed in splenectomized rabbits infected with Eimeria stiedae and in normal and splenectomized rabbits injected with Leishmania enriettii. No positive complement-fixation tests were observed in all four experiments. The observed titre differences between the inactivated and nonactivated sera in rabbits both normal and splenec- 
tomized may be an exhibition of the phenomenon observed by Cathie (1957) in a lesser degree or may be due to experimental errors. Since this difference seems to be more or less constant, the present author holds the former view. Additional evidence for this view comes from similar observations of Sabin and Feldman (1948), Jettmar (1954), Grönroos (1955), and Cathie and Cecil (1957).

The dye test and complement-fixation test titres shown by the guinea-pig infected with Leishmania enriettii is clearly a case of concurrent infection. It is unfortunate that this diagnosis could not be clinched by the isolation of Toxoplasma from this animal. The absence of dye test antibodies in animals inoculated with Atoxoplasma shows that this parasite is immunologically different from Toxoplasma. Crithidia fasciculata did not produce any dye test antibodies as expected.

Summing up, so far no protozoan parasite has been conclusively incriminated as producing dye test antibodies which could be detected by the dye test as described by Sabin and Feldman (1948).

I am greatly indebted to the staff of the North London Blood Transfusion Centre in general and to Dr. P. B. Booth in particular for assistance gladly given in the choice of accessory factor serum donors. Without their help, this work could not have been undertaken. My thanks are also due to Mr. W. Cooper and Mr. P. E. Nesbitt, of this department, for assisting me in the large number of splenectomies carried out and also for preparing the various cultures. I am grateful to my colleagues Drs. B. Dasgupta, M. A. AlDabagh, and H. Prasad for technical assistance in the course of this work. My thanks are also due to Dr. R. Lainson for his assistance and helpful criticism and to Dr. A. Westphal of the Protozoologische Abteilung, Bernhard-Nocht-Institut für Schiffs- und Tropenkrankheiten, Hamburg, for the supply of a photostat copy of one of his early papers. I am gratefully indebted to Professor P. C. C. Garnham for his keen interest and for helpful suggestions and criticisms in the course of this work.

\section{REFERENCES}

Awad, F. I. (1954a). Trans. roy. Soc. trop. Med. Hyg., 48, 337. - (1954b). Lancet, 2, 1055.

- and Lainson, R. (1954a). Ibid., 1, 574.

(1954b). J. clin. Path., 7, 152

Beverley, J. K. A. (1957). Trans. roy. Soc. trop. Med. Hyg., 51, 118. Beattie, C. P., and Roseman, C. (1954). J. Hyg. (Lond.), $52,37$. biol. scand., $\mathbf{4 1}, 361$.

Cathie, I. A. B. (1955a). Proc. roy. Soc. Med., 48, 1074. (1955b). Gt Ormond Str. J., No. 10, 81. (1957). Trans. roy. Soc. trop. Med. Hyg., 51, 104.

and Cecil, G.W. (1957). Lancet, 1, 816 . biologia Roma: Riassuntidelle Comunicazioni Vol 2 di Micro-

Grönroos, P. (1955). Ann. Med. exp. Fenn., 33, 310.

and Salminen, A. (1955). Ibid., 33, 141.

Jacobs, L. (1956). Ann. N.Y. Acad. Sci., 64, 154.

Jettmar, H. M. (1954). Wien. klin. Wschr., 66, 276.

Lainson, R. (1955). Ann. trop. Med. Parasit., $49,384$.

Michalzik, K. (1953). Dtsch. med. Wschr., 78, 307.

Moscovici, C. (1953). In VI. Congresso Internazionale di Microbiologia, Roma: Riassunti delle Comunicazioni, Vol. 2, p. 567.

- (1954). R.C. Ist. sup. Sanit., Roma, 17, 1002.

Mühlpfordt, H. (1951). Z. Tropenmed. Parasit., 3, 205.

Piekarski, G. (1958). C' 'sl. Parasit., 5, 137.

Saathoff, M., and Korte, W.'(1957). Z. Tropenmed. Parasit., $8,356$.

Reid, J. D., and Manning, J. D. (1956). N.Z. med. J., 55, 448.

Sabin, A. B., and Feldman, H. A. (1948). Science, 108, 660.

Thalhammer, O. (1957). Die Toxoplasmose bei Mensch und Tier. Wilhelm Maudrich, Wien.

Thiel, P. H. van (1958). Antonie v. Leeuwenhoek, 24, 113.

Varela, G., Roch, E., and Vázquez, A. (1955). Rev. Inst. Salubr. Enferm. trop. (Méx.), 15, 73 .

Warren, J., and Russ, S. B. (1948). Proc. Soc. exp. Biol. (N.Y.), 67, 85.

Westphal, A., and Knüttgen, H. (1950). Med. Mschr., 4, 196. 